\title{
Event-Based Consensus for General Linear Multiagent Systems under Switching Topologies
}

\author{
Yinshuang Sun $\mathbb{D}^{1}{ }^{1}$ Zhijian Ji $\mathbb{D}^{1},{ }^{1}$ and Kaien Liu $\mathbb{D D}^{2}$ \\ ${ }^{1}$ Institute of Complexity Science, College of Automation, Qingdao University, Qingdao 266071, China \\ ${ }^{2}$ School of Mathematics and Statistics, Qingdao University, Qingdao 266071, China \\ Correspondence should be addressed to Zhijian Ji; jizhijian@pku.org.cn
}

Received 14 December 2019; Revised 21 January 2020; Accepted 3 February 2020; Published 28 March 2020

Academic Editor: Lucia Valentina Gambuzza

Copyright ( $(2020$ Yinshuang Sun et al. This is an open access article distributed under the Creative Commons Attribution License, which permits unrestricted use, distribution, and reproduction in any medium, provided the original work is properly cited.

In this paper, event-triggered leader-following consensus of general linear multiagent systems under both fixed topology and switching topologies is studied. First, centralised and decentralised event-triggered control strategies based on neighbors' state estimation are proposed under fixed topology, in which the controller is only updated at the time of triggering. Obviously, compared with the continuous time control algorithms, the event-triggered control strategies can reduce the communication frequency among agents effectively. Meanwhile, event-triggering conditions are derived for systems to achieve consensus by using the Lyapunov stability theory and model transformation method. Then, the theoretical results obtained under the fixed topology are extended to the switching topologies, and the sufficient conditions for the system to achieve leader-following consensus under the switching topologies are given. However, different from fixed topology, the control input of each agent is updated both at event-triggering and topology switching time. Finally, Zeno behaviors can be excluded by proving that the minimum triggering interval of each agent is strictly positive, and the effectiveness of the event-triggered protocol is verified by simulation experiments.

\section{Introduction}

In recent years, multi-agent systems have great potential of application in the fields of biology, engineering, physics, society and so on. Also its distributed cooperative control [1, 2] has attracted more and more researchers' attention. For example, the controllability [3-11] and the consensus problem [12-19] are widely studied in multi-agent systems. Among them, as the basic problem of multi-agent cooperative control, consensus is widely used in formation control [20], cluster control, sensor network and other aspects, which is a research hot issue in the control discipline at present.

In practical applications, the information needed for cooperative control among agents is transmitted through the network. It is necessary to design a reasonable controller to ensure the control performance of the system due to the finite energy of the agent and the limited network bandwidth. It is well known that periodic sampling control [21-23] can save resources, but when the system runs in an ideal environment or the system state tends to be consensus gradually, it will cause unnecessary resource waste if the control task is executed periodically. In order to reduce this unnecessary waste of resources, a new simple event-triggered control strategy based on feedback mechanism was proposed in [24]. In short, the event-triggered control strategy means that the control task is executed as required. On the premise of ensuring the closed-loop system has certain performance, only can the task be executed once when a specific event occurs (such as the state error exceeds the preset threshold value). The advantage of event-triggered control strategies is that it can not only guarantee the performance of the system but also save the network and computing resources. At present, event-triggered mechanism has been applied to the research of consensus for multiagent systems effectively. For example, Dimarogonas and Frazoli and Dimarogonas et al. $[25,26]$ studied the consensus of a first-order multiagent system in undirected topology, and Yan et al. [27] investigated the consensus of a second-order multiagent system based on event-triggered mechanism in directed topology. Eventtriggering conditions based on composite measurements were 
designed in [28] to address the consensus of a multiagent system. Hu et al. and Li et al. $[29,30]$ discussed the leaderfollowing consensus of the second-order multiagent system. The centralized and decentralized event-triggered strategies were proposed in [31], which made the state of all agents converge to the same value gradually. In [32], a consensus control algorithm based on event-triggered mechanism was proposed for the nonlinear multiagent system. In [33], a new average consensus problem under event-triggered control strategy was set up, which limits the measurement error of each agent to a threshold that changes with time. Meng and Chen [34] studied the average consensus of the event-triggered multi-integrator systems under fixed and switching topologies and designed an event-triggered scheme based on quadratic Lyapunov function, which made each agent's state converge to it's initial average eventually. Xiao et al. [35] discussed the average consensus of the network of the integrator system with unidirectional information link. In order to reduce the communication cost, a distributed state consensus sampling data control scheme based on edge-event was proposed. In recent years, more and more attention has been paid to general linear systems. In [36], the consensus of general linear multiagent systems under integral event-triggered strategy has been considered. In [37], the consensus of general linear systems under fixed topology and switching topologies has been investigated. Hu et al. [38] studied the leader-following consensus of general linear multiagent system under fixed topology, and Zhu et al. [39] studied the consensus of general linear multiagent system under mixed event-triggering conditions.

With this background, we consider the event-triggered leader-following consensus of the general linear multiagent system under fixed topology and switching topologies. Event-triggered control mechanism is designed for each agent, respectively. Under the mechanism, multiagent systems can achieve leader-following consensus gradually. Moreover, a continuous event-triggering condition is proposed, which uses the state error between the follower and leader to design the triggering conditions of each agent under the fixed topology and switching topologies, respectively. The consensus problem is transformed into the stability problem by the method of model transformation, and the sufficient conditions for the system to achieve leader-following consensus are obtained by using Lyapunov stability theory. In addition, all the proposed event-triggered mechanism can exclude Zeno behavior. Finally, the accuracy of the conclusion is verified by simulation experiment.

The structure of this paper is as follows. Section 2 introduces some concepts of the graph theory and system model. The leader-following consensus of the systems under the fixed/switching topologies is considered in Sections 3 and 4 In addition, the effectiveness of the results is shown through simulation experiment in Section 5. Section 6 summarizes this paper.

The following notations are used in this paper. $\otimes$ denotes the Kronecker product. $I_{N}$ denotes the $N$-dimensional identity matrix. $\mathbb{R}^{n}$ and $\mathbb{R}^{m \times n}$ indicate the set of $n$ dimensional real vectors and $m \times n$ dimensional real matrices, respectively.

\section{Preliminaries}

2.1. Theory of Graph. For a multiagent system composed of a leader and follower agents, its communication topology can be represented by an undirected graph $\overline{\mathscr{G}}=(\overline{\mathscr{V}}, \bar{E})$, where $\overline{\mathscr{V}}=\{0,1, \ldots, N\} .0$ denotes the leader, and $1, \ldots, N$ denote the followers. $\bar{E} \subseteq \overline{\mathscr{V}} \times \overline{\mathscr{V}}$ denotes the edges set. The connection matrix between the follower agent $i(i=1, \ldots, N)$ and leader 0 is $\bar{D}=\operatorname{diag}\left\{a_{10}, \ldots, a_{N 0}\right\}$, where $a_{i 0}$ is the connection weight between the leader 0 and follower $i$. If $a_{i 0}>0$, the follower agent $i$ can receive state information of leader 0; otherwise, $a_{i 0}=0$.

The communication network among the followers is denoted by $\mathscr{G}=(\mathscr{V}, E, A)$, where $\mathscr{V}=\{1,2, \ldots, N\}$ and $E \subseteq \mathscr{V} \times \mathscr{V}$ are obtained from $\bar{E}$ by removing all edges among the leader 0 and followers in $\overline{\mathscr{V}}$, and $A=\left(a_{i j}\right) \in \mathbb{R}^{N \times N}$ is the weighted adjacency matrix of graph $\mathscr{G}$, where $a_{i j}>0$ for $(i, j) \in E$ if agent $i$ obtains information from agent $j$. We assume that $(i, i) \notin E$, and hence $a_{i i}=0$. For a given graph $\mathscr{G}$ with the adjacency matrix $A$, the Laplacian matrix used in this paper is $L=D-A$, where $D$ is a diagonal matrix, its diagonal elements are $d_{i i}=\sum_{j \in N_{i}}\left|a_{i j}\right|$, and define $N_{i}$ as the neighbor set of agent $i$ in $\mathscr{V}$. Therefore, the elements of $L$ are

$$
L_{i k}= \begin{cases}\sum_{j \in N_{i}}\left|a_{i j}\right|, & k=i, \\ -a_{i k}, & k \neq i .\end{cases}
$$

A path from the vertex $i$ to vertex $k$ is a sequence of adjacent edges in the form $(i, i+1),(i+1, i+2), \ldots$, $(k-1, k)$. The undirected graph is said to be connected if there exists a path between any two distinct vertices.

2.2. System Model. Consider a multiagent system composed of the leader 0 and $N$ followers. The dynamics of leader 0 is

$$
\dot{x}_{0}(t)=A x_{0}(t),
$$

where $x_{0}(t) \in \mathbb{R}^{n}$ is the state and $A \in \mathbb{R}^{n \times n}$ is constant matrix.

Accordingly, each follower has the following linear dynamic equation:

$$
\dot{x}_{i}(t)=A x_{i}(t)+B u_{i}(t), \quad i=1, \ldots, N,
$$

where $x_{i}(t) \in \mathbb{R}^{n}$ and $u_{i}(t) \in \mathbb{R}^{p}$ are the state and input of the $i$ th follower agent, respectively. $A \in \mathbb{R}^{n \times n}$ and $B \in \mathbb{R}^{n \times p}$ are constant matrices. Denote the initial state of the $i$ th follower as $x_{i}(0)$.

Definition 1. If there is a control input $u_{i}(t)$, the leader 0 and follower $i$ for any initial state satisfy the following conditions:

$$
\lim _{t \longrightarrow \infty}\left\|x_{i}(t)-x_{0}(t)\right\|=0, \quad i=1,2, \ldots, N .
$$

Then, the leader (2) is said to be successfully tracked by follower (3).

Assumption 1. The communication network topology $\mathscr{G}$ among followers is connected. 
Assumption 2. The pair $(A, B)$ is stabilizable.

Based on Assumption 2, there is a symmetric positive definite matrix $P$ that satisfies the following algebraic Riccati and Lyapunov inequality with $\beta>0$ :

$$
\begin{array}{r}
A^{T} P+P A-2 \beta P B B^{T} P+\beta I<0, \\
A^{T} P+P A<0 .
\end{array}
$$

Lemma 1 (see [37]). For an undirected and connected graph $\mathscr{G}$, the eigenvalues of $L$ are real and can be labelled as

$$
0=\lambda_{1}(L)<\lambda_{2}(L) \leq \cdots \leq \lambda_{N}(L) .
$$

Lemma 2 (see [40]). For any $x, y \in \mathbb{R}$ and $\beta>0$, one has the following property:

$$
x y \leq \frac{\beta}{2} x^{2}+\frac{1}{2 \beta} y^{2}
$$

Lemma 3 (see [41]). (Comparison Principle). Consider a differential equation $(d u / d t)=f(t, u), u\left(t_{0}\right)=u_{0}$, where $t>0, f(t, u)$ is continuous and satisfies the local Lipschitz condition in $t$. Let $\left[t_{0}, T\right)$ be the maximum existence interval of the solution $u$, where Tcan be infinite. If, for any $t \in\left[t_{0}, T\right)$ satisfies

$$
\begin{aligned}
\frac{\mathrm{d} v}{\mathrm{~d} t} & \leq f(t, v), \\
v\left(t_{0}\right) & \leq u_{0},
\end{aligned}
$$

then $v(t) \leq u(t), t \in\left[t_{0}, T\right)$.

\section{Leader-Following Control of Multiagent Systems under Fixed Topology}

In this part, we consider the leader-following control of multiagent systems (2) and (3) under the event-triggered strategy. Based on the general event-triggered control law, we put forward two kinds of piecewise continuous control mechanisms, which are centralized event-triggered mechanism and decentralized event-triggered mechanism with state estimation in order to minimize the frequency of controller updating. The analysis shows that under the two control mechanisms, multiagent system (3) can track the system (2) successfully with appropriate event-triggering function. The minimum interval between any two consecutive event-triggering instants under the two control mechanisms is greater than 0 , and Zeno behavior can be excluded.

3.1. Centralized Event-Triggered Control Strategy. Under the centralized event-triggered strategy, all agents $i$ in system (3) are triggered synchronously at the time $t_{k}(k=0,1, \ldots$,$) . At$ the triggering instants, all agents send their states information to neighbours and update the control law with the received state information. Compared with the control protocol in continuous time, each agent $i$ only updates the control input at the event instants under the event-triggered mechanism. So, $u_{i}$ is a piecewise continuous function, and the updating frequency can be reduced.

We consider the following control input for the $i$ th follower:

$$
\begin{aligned}
u_{i}(t)= & -K \sum_{j \in N_{i}(t)} a_{i j}(t)\left(x_{i}\left(t_{k}\right)-x_{j}\left(t_{k}\right)\right) \\
& -K a_{i 0}(t)\left(x_{i}\left(t_{k}\right)-x_{0}\left(t_{k}\right)\right)
\end{aligned}
$$

where $t \in\left[t_{k}, t_{k+1}\right), K \in \mathbb{R}^{p \times n}$ is the control gain matrix to be designed, and $x_{i}\left(t_{k}\right)$ is the sampling state of agent $i$ at the $k$ th triggering instant. Since there does not exist control input for leader 0 , we take $x_{0}\left(t_{k}\right)=x_{0}(t), t \in\left[t_{k}, t_{k+1}\right)$. For convenience, we make $t_{0}=0$.

The event-triggering time sequence $\left\{t_{k}\right\}$ is determined by the following triggering functions:

$$
\begin{aligned}
f(t)= & -\kappa \frac{a_{i 0_{\min }} \lambda_{\min }(W)-\alpha \lambda_{\max }(P)}{a_{i 0_{\max }} \lambda_{\max }(W)+2 \lambda_{\max }(D) \lambda_{\max }(W)} \sum_{i=1}^{N} \widehat{x}_{i}^{T} \widehat{x}_{i} \\
& +\sum_{i=1}^{N} e_{i}^{T} e_{i} \geq 0 .
\end{aligned}
$$

That is, $t_{k+1}=\inf \left\{t>t_{k} \mid f(t) \geq 0\right\}$, where $0<\kappa<1,0<$ $\alpha<\left(a_{i 0_{\min }} \lambda_{\min }(W) / \lambda_{\max }(P)\right), W=P B B^{T} P$.

The state error between the follower and leader is defined as $\hat{x}_{i}(t)=x_{i}(t)-x_{0}(t), \hat{x}(t)=\left[\hat{x}_{1}^{T}, \ldots, \hat{x}_{N}^{T}\right]^{T}$. For agent $i$, the measurement error is defined as $e_{i}(t)=x_{i}\left(t_{k}\right)-$ $x_{i}(t), t \in\left[t_{k}, t_{k+1}\right)$. Then, formula (10) is converted to

$$
\begin{aligned}
u_{i}(t)= & -K \sum_{j=0}^{N} a_{i j}\left(\hat{x}_{i}(t)-\hat{x}_{j}(t)+e_{i}(t)-e_{j}(t)\right) \\
= & -K\left(\sum_{j=1}^{N} a_{i j}\left(\hat{x}_{i}(t)-\hat{x}_{j}(t)+e_{i}(t)-e_{j}(t)\right)\right) \\
& -K a_{i 0}\left(\hat{x}_{i}(t)+e_{i}(t)\right) .
\end{aligned}
$$

Combining (2), (3), and (12), we get

$$
\begin{aligned}
\dot{\hat{x}}(t) & =\left(I_{N} \otimes A\right) \hat{x}(t)-\left(I_{N} \otimes B K\right)\left((L+\bar{D}) \otimes I_{n}\right)(\widehat{x}(t)+e(t)) \\
& =\left(I_{N} \otimes A\right) \hat{x}(t)-((L+\bar{D}) \otimes B K)(\widehat{x}(t)+e(t)) .
\end{aligned}
$$

Remark 1. Through the model transformation, the leaderfollowing control problem between systems (2) and (3) can be interpreted by the stability problem of system (13).

Next, we will give the following consensus conditions under the centralized event-triggering protocol (10).

Theorem 1. Under Assumptions 1 and 2, centralized eventtriggered control strategy (10) can make multiagent system (3) track system (2) successfully under event-triggering condition (4), where feedback gain matrix $K$ satisfies $K=B^{T} P$ and $W=P B K$. 
Proof. We consider a candidate Lyapunov function, as follows:

$$
V_{1}=e^{\alpha t} \sum_{i=1}^{N} \widehat{x}_{i}^{T} P \widehat{x}_{i}
$$

Along with the trajectories of the state as described in (8), the time derivative of Lyapunov function is

$$
\begin{aligned}
\dot{V}_{1} & =\alpha e^{\alpha t} \sum_{i=1}^{N} \widehat{x}_{i}^{T} P \widehat{x}_{i}+2 e^{\alpha t} \sum_{i=1}^{N} \widehat{x}_{i}^{T} P \dot{\hat{x}}_{i} \\
& =e^{\alpha t} \sum_{i=1}^{N} \widehat{x}_{i}^{T} \alpha P \widehat{x}_{i}+2 e^{\alpha t} \sum_{i=1}^{N} \widehat{x}_{i}^{T} P\left(A \widehat{x}_{i}+B u_{i}\right) \\
& =e^{\alpha t} \sum_{i=1}^{N} \widehat{x}_{i}^{T} \alpha P \widehat{x}_{i}+2 e^{\alpha t} \sum_{i=1}^{N} \widehat{x}_{i}^{T} P A \widehat{x}_{i}+2 e^{\alpha t} \sum_{i=1}^{N} \widehat{x}_{i}^{T} P B u_{i},
\end{aligned}
$$

where

$$
\begin{aligned}
e^{\alpha t} \sum_{i=1}^{N} \widehat{x}_{i}^{T} P B u_{i} \\
=-e^{\alpha t} \sum_{i=1}^{N} \hat{x}_{i}^{T} P B K\left(a_{i 0}\left(\widehat{x}_{i}+e_{i}\right)+\sum_{j=1}^{N} a_{i j}\left(\hat{x}_{i}-\hat{x}_{j}+e_{i}-e_{j}\right)\right) \\
=-e^{\alpha t} \sum_{i=1}^{N} \hat{x}_{i}^{T} W a_{i 0}\left(\hat{x}_{i}+e_{i}\right)-e^{\alpha t} \sum_{i=1}^{N} \hat{x}_{i}^{T} W \sum_{j=1}^{N} a_{i j}\left(\hat{x}_{i}-\widehat{x}_{j}\right) \\
\quad-e^{\alpha t} \sum_{i=1}^{N} \widehat{x}_{i}^{T} W \sum_{j=1}^{N} a_{i j}\left(e_{i}-e_{j}\right),
\end{aligned}
$$

where $W=P B K$.

According to the property of $L=L^{T}$ in undirected graph $\mathscr{G}$, we can deduce

$$
\begin{aligned}
& e^{\alpha t} \sum_{i=1}^{N} \widehat{x}_{i}^{T} W \sum_{j=1}^{N} a_{i j}\left(\widehat{x}_{i}-\widehat{x}_{j}\right) \\
& =e^{\alpha t} \sum_{i=1}^{N} \sum_{j=1}^{N} a_{i j} \hat{x}_{i}^{T} W\left(\hat{x}_{i}-\widehat{x}_{j}\right) \\
& =e^{\alpha t} \sum_{i=1}^{N} \sum_{j=1}^{N} a_{j i} \widehat{x}_{j}^{T} W\left(\hat{x}_{j}-\widehat{x}_{i}\right) \\
& =-e^{\alpha t} \sum_{i=1}^{N} \sum_{j=1}^{N} a_{i j} \widehat{x}_{j}^{T} W\left(\hat{x}_{i}-\widehat{x}_{j}\right) \\
& =\frac{1}{2} e^{\alpha t} \sum_{i=1}^{N} \sum_{j=1}^{N} a_{i j}\left(\hat{x}_{i}-\widehat{x}_{j}\right)^{T} W\left(\widehat{x}_{i}-\widehat{x}_{j}\right) .
\end{aligned}
$$

Similarly,

$$
\begin{aligned}
e^{\alpha t} & \sum_{i=1}^{N} \widehat{x}_{i}^{T} W \sum_{j=1}^{N} a_{i j}\left(e_{i}-e_{j}\right) \\
& =\frac{1}{2} e^{\alpha t} \sum_{i=1}^{N} \sum_{j=1}^{N} a_{i j}\left(\hat{x}_{i}-\widehat{x}_{j}\right)^{T} W\left(e_{i}-e_{j}\right) .
\end{aligned}
$$

Hence,

$$
\begin{aligned}
& e^{\alpha t} \sum_{i=1}^{N} \widehat{x}_{i}^{T} P B u_{i} \\
& =-e^{\alpha t} \sum_{i=1}^{N} \widehat{x}_{i}^{T} W a_{i 0}\left(\widehat{x}_{i}+e_{i}\right)-\frac{1}{2} e^{\alpha t} \sum_{i=1}^{N} \sum_{j=1}^{N} a_{i j}\left(\widehat{x}_{i}-\widehat{x}_{j}\right)^{T} W\left(\widehat{x}_{i}-\widehat{x}_{j}\right) \\
& \quad-\frac{1}{2} e^{\alpha t} \sum_{i=1}^{N} \sum_{j=1}^{N} a_{i j}\left(\widehat{x}_{i}-\widehat{x}_{j}\right)^{T} W\left(e_{i}-e_{j}\right) \\
& =-e^{\alpha t} \sum_{i=1}^{N} \widehat{x}_{i}^{T} W a_{i 0}\left(\widehat{x}_{i}+e_{i}\right)-e^{\alpha t} \sum_{i=1}^{N} \sum_{j=1}^{N} a_{i j} \widehat{x}_{i}^{T} W\left(\widehat{x}_{i}-\widehat{x}_{j}\right) \\
& \quad-\frac{1}{2} e^{\alpha t} \sum_{i=1}^{N} \sum_{j=1}^{N} a_{i j}\left(\widehat{x}_{i}-\widehat{x}_{j}\right)^{T} W\left(e_{i}-e_{j}\right) .
\end{aligned}
$$

Combining equality (15) yields

$$
\begin{aligned}
\dot{V}_{1}= & e^{\alpha t} \sum_{i=1}^{N} \widehat{x}_{i}^{T} \alpha P \widehat{x}_{i}+2 e^{\alpha t} \sum_{i=1}^{N} \widehat{x}_{i}^{T} P A \widehat{x}_{i} \\
& -2 e^{\alpha t} \sum_{i=1}^{N} \sum_{j=1}^{N} a_{i j} \widehat{x}_{i}^{T} W\left(\widehat{x}_{i}-\widehat{x}_{j}\right) \\
& -e^{\alpha t} \sum_{i=1}^{N} \sum_{j=1}^{N} a_{i j}\left(\widehat{x}_{i}-\widehat{x}_{j}\right)^{T} W\left(e_{i}-e_{j}\right) \\
& -2 e^{\alpha t} \sum_{i=1}^{N} \hat{x}_{i}^{T} W a_{i 0}\left(\widehat{x}_{i}+e_{i}\right) .
\end{aligned}
$$

In the light of Lemma 2, we have

$$
\begin{aligned}
& -e^{\alpha t} \sum_{i=1}^{N} \sum_{j=1}^{N} a_{i j}\left(\widehat{x}_{i}-\widehat{x}_{j}\right)^{T} W\left(e_{i}-e_{j}\right) \\
& \leq \frac{1}{2} e^{\alpha t} \sum_{i=1}^{N} \sum_{j=1}^{N} a_{i j}\left(\hat{x}_{i}-\widehat{x}_{j}\right)^{T} W\left(\hat{x}_{i}-\hat{x}_{j}\right)+ \\
& \frac{1}{2} e^{\alpha t} \sum_{i=1}^{N} \sum_{j=1}^{N} a_{i j}\left(e_{i}-e_{j}\right)^{T} W\left(e_{i}-e_{j}\right) .
\end{aligned}
$$

By substituting the abovementioned formula into equation (20), we obtain 


$$
\begin{aligned}
& \dot{V}_{1} \leq e^{\alpha t} \sum_{i=1}^{N} \widehat{x}_{i}^{T} \alpha P \widehat{x}_{i}+2 e^{\alpha t} \sum_{i=1}^{N} \widehat{x}_{i}^{T} P A \widehat{x}_{i} \\
& -2 e^{\alpha t} \sum_{i=1}^{N} \sum_{j=1}^{N} a_{i j} \hat{x}_{i}^{T} W\left(\hat{x}_{i}-\hat{x}_{j}\right) \\
& +\frac{e^{\alpha t}}{2} \sum_{i=1}^{N} \sum_{j=1}^{N} a_{i j}\left(\hat{x}_{i}-\hat{x}_{j}\right)^{T} W\left(\hat{x}_{i}-\hat{x}_{j}\right) \\
& +\frac{e^{\alpha t}}{2} \sum_{i=1}^{N} \sum_{j=1}^{N} a_{i j}\left(e_{i}-e_{j}\right)^{T} W\left(e_{i}-e_{j}\right) \\
& -2 e^{\alpha t} \sum_{i=1}^{N} \widehat{x}_{i}^{T} W a_{i 0}\left(\widehat{x}_{i}+e_{i}\right) \\
& \leq e^{\alpha t} \sum_{i=1}^{N} \widehat{x}_{i}^{T} \alpha P \widehat{x}_{i}+2 e^{\alpha t} \sum_{i=1}^{N} \widehat{x}_{i}^{T} P A \widehat{x}_{i} \\
& -2 e^{\alpha t} \sum_{i=1}^{N} \sum_{j=1}^{N} a_{i j} \widehat{x}_{i}^{T} W\left(\widehat{x}_{i}-\widehat{x}_{j}\right) \\
& +e^{\alpha t} \sum_{i=1}^{N} \sum_{j=1}^{N} a_{i j} \hat{x}_{i}^{T} W\left(\hat{x}_{i}-\widehat{x}_{j}\right) \\
& \dot{V}_{1} \leq e^{\alpha t} \sum_{i=1}^{N} \widehat{x}_{i}^{T} \alpha P \widehat{x}_{i}+2 e^{\alpha t} \sum_{i=1}^{N} \widehat{x}_{i}^{T} P A \widehat{x}_{i} \\
& -e^{\alpha t} \sum_{i=1}^{N} \sum_{j=1}^{N} a_{i j} \widehat{x}_{i}^{T} W\left(\widehat{x}_{i}-\widehat{x}_{j}\right) \\
& +2 e^{\alpha t} \sum_{i=1}^{N} \sum_{j=1}^{N} a_{i j} e_{i}^{T} W e_{i} \\
& -2 e^{\alpha t} \sum_{i=1}^{N} \hat{x}_{i}^{T} W a_{i 0}\left(\hat{x}_{i}+e_{i}\right) \\
& -e^{\alpha t} \sum_{i=1}^{N} \widehat{x}_{i}^{T} W a_{i 0}\left(\widehat{x}_{i}+e_{i}\right) \\
& =-e^{\alpha t} \sum_{i=1}^{N} \hat{x}_{i}^{T} W a_{i 0} \widehat{x}_{i}-e^{\alpha t} \sum_{i=1}^{N} \hat{x}_{i}^{T} W a_{i 0} e_{i} \\
& \leq-e^{\alpha t} \sum_{i=1}^{N} \widehat{x}_{i}^{T} W a_{i 0} \widehat{x}_{i}+\frac{1}{2} e^{\alpha t} \sum_{i=1}^{N} \widehat{x}_{i}^{T} W a_{i 0} \widehat{x}_{i} \\
& +\frac{1}{2} e^{\alpha t} \sum_{i=1}^{N} e_{i}^{T} W a_{i 0} e_{i} \\
& \leq-\frac{1}{2} e^{\alpha t} \sum_{i=1}^{N} \widehat{x}_{i}^{T} W a_{i 0} \widehat{x}_{i}+\frac{1}{2} e^{\alpha t} \sum_{i=1}^{N} e_{i}^{T} W a_{i 0} e_{i} .
\end{aligned}
$$$$
+\frac{e^{\alpha t}}{2} \sum_{i=1}^{N} \sum_{j=1}^{N} a_{i j}\left(e_{i}-e_{j}\right)^{T} W\left(e_{i}-e_{j}\right)
$$$$
-2 e^{\alpha t} \sum_{i=1}^{N} \hat{x}_{i}^{T} W a_{i 0}\left(\hat{x}_{i}+e_{i}\right)
$$$$
\leq e^{\alpha t} \sum_{i=1}^{N} \widehat{x}_{i}^{T} \alpha P \widehat{x}_{i}+2 e^{\alpha t} \sum_{i=1}^{N} \widehat{x}_{i}^{T} P A \widehat{x}_{i}
$$$$
-e^{\alpha t} \sum_{i=1}^{N} \sum_{j=1}^{N} a_{i j} \widehat{x}_{i}^{T} W\left(\widehat{x}_{i}-\widehat{x}_{j}\right)
$$$$
+\frac{e^{\alpha t}}{2} \sum_{i=1}^{N} \sum_{j=1}^{N} a_{i j}\left(e_{i}-e_{j}\right)^{T} W\left(e_{i}-e_{j}\right)
$$$$
-2 e^{\alpha t} \sum_{i=1}^{N} \widehat{x}_{i}^{T} W a_{i 0}\left(\hat{x}_{i}+e_{i}\right) .
$$

Combining (24) and (25), we arrive at

$$
\begin{aligned}
\dot{V}_{1} \leq & e^{\alpha t} \sum_{i=1}^{N} \widehat{x}_{i}^{T} \alpha P \widehat{x}_{i}+2 e^{\alpha t} \sum_{i=1}^{N} \widehat{x}_{i}^{T} P A \widehat{x}_{i} \\
& -e^{\alpha t} \sum_{i=1}^{N} \sum_{j=1}^{N} a_{i j} \widehat{x}_{i}^{T} W\left(\widehat{x}_{i}-\widehat{x}_{j}\right) \\
& -e^{\alpha t} \sum_{i=1}^{N} \widehat{x}_{i}^{T} W a_{i 0} \widehat{x}_{i}+e^{\alpha t} \sum_{i=1}^{N} e_{i}^{T} W a_{i 0} e_{i} \\
& +2 e^{\alpha t} \sum_{i=1}^{N} \sum_{j=1}^{N} a_{i j} e_{i}^{T} W e_{i} \\
\leq & e^{\alpha t} \widehat{x}^{T}\left(I_{N} \otimes \alpha P\right) \widehat{x}+e^{\alpha t} \widehat{x}^{T}\left(I_{N} \otimes\left(P A+A^{T} P\right)\right) \hat{x} \\
& -e^{\alpha t} \widehat{x}^{T}(L \otimes W) \widehat{x}-e^{\alpha t} \widehat{x}^{T}(\bar{D} \otimes W) \widehat{x} \\
& +e^{\alpha t} e^{T}(\bar{D} \otimes W) e+2 e^{\alpha t} e^{T}(D \otimes W) e .
\end{aligned}
$$

Under Assumption 1, by using Lemma $1, \widehat{x}^{T}(L \otimes W) \widehat{x} \geq$ $\lambda_{2}(L) \hat{x}^{T}\left(I_{N} \otimes W\right) \widehat{x}$ holds. Consequently,

$$
\begin{aligned}
\dot{V}_{1} \leq & e^{\alpha t} \widehat{x}^{T}\left(I_{N} \otimes\left(P A+A^{T} P-\lambda_{2}(L) W\right)\right) \hat{x} \\
& +e^{\alpha t}\left(\alpha \lambda_{\max }(P)-a_{i 0_{\min }} \lambda_{\min }(W)\right)\|\hat{x}\|^{2} \\
& +e^{\alpha t}\left(a_{i 0_{\max }} \lambda_{\max }(W)+2 \lambda_{\max }(D) \lambda_{\max }(W)\right)\|e\|^{2} .
\end{aligned}
$$

Using inequality (5) and event-triggering condition (11), we claim that the following inequality holds: 


$$
\begin{aligned}
\dot{V}_{1} \leq & (\kappa-1) e^{\alpha t}\left(a_{i 0_{\min }} \lambda_{\min }(W)-\alpha \lambda_{\max }(P)\right)\|\widehat{x}\|^{2} \\
& -e^{\alpha t} \frac{\lambda_{2}(L)}{2} \widehat{x}^{T} \widehat{x} \leq-e^{\alpha t} \frac{\lambda_{2}(L)}{2} \widehat{x}^{T} \widehat{x} .
\end{aligned}
$$

It can be seen from (28) that $V_{1}$ is not increasing; therefore,

$$
V_{1}(0) \geq V_{1}(t)=e^{\alpha t} \sum_{i=1}^{N} \widehat{x}_{i}(t)^{T} P \widehat{x}_{i}(i) \geq e^{\alpha t} \lambda_{\min }(P)\|\widehat{x}(t)\|^{2} .
$$

That is to say, $\|\widehat{x}(t)\| \leq \sqrt{\left(V_{1}(0) / \lambda_{\min }(P)\right)} e^{-(\alpha / 2) t}$, i.e., $\lim _{t \rightarrow \infty} \widehat{x}(t)=0$ is equivalent to $\lim _{t \rightarrow \infty}\left\|\widehat{x}_{i}(t)\right\|=0$, which means $\quad \lim _{t \longrightarrow \infty}\left\|x_{i}(t)-x_{0}(t)\right\|=0, i=1,2, \ldots, N$ holds.

Theorem 2. Under the conditions of Theorem 1, system (13) does not exhibit Zeno behavior. The interval between any two consecutive event-triggering instants of the system is not less than

$$
\frac{\left(\left\|I_{N} \otimes A\right\|+\|(L+\bar{D}) \otimes B K\|\right)}{3} \times\left(\left(1+\sqrt{\frac{\kappa\left(a_{i 0_{\min }} \lambda_{\min }(W)-\alpha \lambda_{\max }(P)\right)}{a_{i 0_{\max }} \lambda_{\max }(W)+2 \lambda_{\max }(D) \lambda_{\max }(W)}}\right)^{3}-1\right) .
$$

Proof. From the mechanism of event-triggering strategy, the event interval between $t_{k}$ and $t_{k+1}$ is the time that $\quad(\|e(t)\| /\|\widehat{x}(t)\|)$ grows from 0 to

$\sqrt{\left(\kappa\left(a_{i 0_{\min }} \lambda_{\min }(W)-\alpha \lambda_{\max }(P)\right) /\left(a_{i 0_{\max }} \lambda_{\max }(W)+2 \lambda_{\max }(D) \lambda_{\max }(W)\right)\right)}$. The time derivative of $(\|e(t)\| /\|\widehat{x}(t)\|)$ has

$$
\begin{aligned}
& \frac{\mathrm{d}}{\mathrm{d} t} \frac{\|e(t)\|}{\|\widehat{x}(t)\|}=\frac{\mathrm{d}}{\mathrm{d} t} \frac{\left(e(t)^{T} e(t)\right)^{1 / 2}}{\left(\widehat{x}(t)^{T} \widehat{x}(t)\right)^{1 / 2}} \\
& =\frac{\left(e(t)^{T} e(t)\right)^{(1 / 2)^{\prime}}\|\widehat{x}(t)\|-\left(e(t)^{T} e(t)\right)^{1 / 2}\left(\widehat{x}(t)^{T} \hat{x}(t)\right)^{(1 / 2)^{\prime}}}{\|\widehat{x}(t)\|^{2}} \\
& =\frac{e(t)^{T} \dot{e}(t)}{\|\widehat{x}(t)\|\|e(t)\|}-\frac{\|e(t)\| \widehat{x}(t)^{T} \dot{\hat{x}}(t)}{\|\widehat{x}(t)\|^{2}\|\widehat{x}(t)\|} \\
& =\frac{-e(t)^{T} \dot{\hat{x}}(t)}{\|\hat{x}(t)\|\|e(t)\|}-\frac{\|e(t)\| \widehat{x}(t)^{T} \dot{\hat{x}}(t)}{\|\hat{x}(t)\|^{2}\|\widehat{x}(t)\|} \\
& \leq \frac{\|\dot{\hat{x}}(t)\|}{\|\widehat{x}(t)\|}+\frac{\|\dot{\hat{x}}(t)\|\|e(t)\|}{\|\hat{x}(t)\|^{2}}=\frac{\|\dot{\hat{x}}(t)\|}{\|\hat{x}(t)\|}\left(1+\frac{\|e(t)\|}{\|\hat{x}(t)\|}\right) \\
& \leq\left(\left\|I_{N} \otimes A\right\|+\|(L+\bar{D}) \otimes B K\|\right)\left(1+\frac{\|e(t)\|}{\|\hat{x}(t)\|}\right) \\
& +\frac{\|(L+\bar{D}) \otimes B K\|\|e\|}{\|\widehat{x}(t)\|}\left(1+\frac{\|e(t)\|}{\|\hat{x}(t)\|}\right) \\
& \leq\left(\left\|I_{N} \otimes A\right\|+\|(L+\bar{D}) \otimes B K\|\right)\left(1+\frac{\|e(t)\|}{\|\widehat{x}(t)\|}\right) \\
& +\frac{\left(\left\|I_{N} \otimes A\right\|+\|(L+\bar{D}) \otimes B K\|\right)\|e\|}{\|\hat{x}(t)\|}\left(1+\frac{\|e(t)\|}{\|\widehat{x}(t)\|}\right) \\
& =\left(\left\|I_{N} \otimes A\right\|+\|(L+\bar{D}) \otimes B K\|\right)\left(1+\frac{\|e(t)\|}{\|\widehat{x}(t)\|}\right)^{2} .
\end{aligned}
$$


Denote $z=(\|e(t)\| /\|\widehat{x}(t)\|)$, then

$$
\dot{z} \leq\left(\left\|I_{N} \otimes A\right\|+\|(L+\bar{D}) \otimes B K\|\right)(1+z)^{2} .
$$

Consider that a nonnegative function $\psi\left(t, \psi_{0}\right)$ satisfies $\dot{\psi}=\left(\left\|I_{N} \otimes A\right\|+\|(L+\bar{D}) \otimes B K\|\right)(1+\psi)^{2}, \quad$ and $\quad \psi_{0}=0$. Then, from Lemma $3, z \leq \psi(t, 0)$. It can be seen from (11) that

$$
\psi(\tau, 0)=\sqrt{\frac{\kappa\left(a_{i 0_{\min }} \lambda_{\min }(W)-\alpha \lambda_{\max }(P)\right)}{a_{i 0_{\max }} \lambda_{\max }(W)+2 \lambda_{\max }(D) \lambda_{\max }(W)}} .
$$

Therefore,

$$
\begin{aligned}
& \tau=\frac{\left(\left\|I_{N} \otimes A\right\|+\|(L+\bar{D}) \otimes B K\|\right)}{3}\left((1+\psi(\tau))^{3}-1\right) \\
& =\frac{\left(\left\|I_{N} \otimes A\right\|+\|(L+\bar{D}) \otimes B K\|\right)}{3} \times\left(\left(1+\sqrt{\left.\frac{\kappa\left(a_{i 0_{\min }} \lambda_{\min }(W)-\alpha \lambda_{\max }(P)\right)}{a_{i 0_{\max } \lambda_{\max }(W)+2 \lambda_{\max }(D) \lambda_{\max }(W)}}\right)^{3}-1}\right) .\right.
\end{aligned}
$$

Obviously, $\tau>0$.

It is assumed that the Zeno behavior occurs, which means that there exists a positive constant $t^{*}$ such that $\lim _{k \rightarrow \infty} t_{k}=t^{*}$. Let $\varepsilon_{0}=(1 / 2) \tau$. There exists a positive integer $N_{0}$ such that $t^{*}-\varepsilon_{0} \leq t_{k} \leq t^{*}$ for the abovementioned $\varepsilon_{0}>0$ according to the definition of sequence limit, where $k \geq N_{0}$. Therefore, $t^{*}+\varepsilon_{0} \leq t_{k}+2 \varepsilon_{0} \leq t_{k+1}$ holds when $k \geq N_{0}$. This contradicts with $t^{*} \geq t_{k+1}$ for $k \geq N_{0}$. Thus, Zeno behavior is strictly excluded.

3.2. Decentralized Event-Triggered Control Strategy. The centralized event-triggered mechanism given in the previous section sets a global state error threshold for all agents. Once the system error reaches the threshold, all agents in the system perform control tasks at the same time. In this section, an error threshold based on the state of its neighbor node is set for each agent. When the state error of the agent reaches the set threshold, the agent triggers the event independently and executes the control task.

The triggering time of the $k$ th event of the $i$ agent is defined as $t_{k}^{i}(k=0,1, \ldots)$. In the design of this section, it should be noted that the agent triggers asynchronously, that is, each agent has its own event-triggering sequence. The measurement error of agent $i$ is defined as $e_{i}(t)=$ $x_{i}\left(t_{k}^{i}\right)-x_{i}(t), t \in\left[t_{k}^{i}, t_{k+1}^{i}\right)$. It is clear that $e_{i}\left(t_{k}^{i}\right)=0$ when $t=t_{k}^{i}$.

For a multiagent system composed of (2) and (3), we consider the following decentralized event-triggered control protocol:

$$
\begin{aligned}
u_{i}(t)= & -K \sum_{j \in N_{i}(t)} a_{i j}(t)\left(x_{i}\left(t_{k}^{i}\right)-x_{j}\left(t_{k^{\prime}}^{i}\right)\right) \\
& -K a_{i 0}(t)\left(x_{i}\left(t_{k}^{i}\right)-x_{0}(t)\right),
\end{aligned}
$$

where $t \in\left[t_{k^{\prime}}^{i}, t_{k+1}^{i}\right), t_{k^{\prime}}^{j}=\arg \min _{l \in N, t \geq t_{l}^{j}}\left\{t-t_{l}^{j}\right\}$ represents the latest event-triggering time before $t$ for agent $j$. According to (35), agent $i$ will update control input $u_{i}$ at both its triggering instants $\left(t_{0}^{i}, t_{1}^{i}, \ldots\right)$ and neighbor agent $j$ event instants $\left(t_{0}^{j}, t_{1}^{j}, \ldots\right)$. The event-triggering instant sequence $\left\{t_{k}^{i}\right\}$ for agent $i$ is determined by the following decentralized event-triggering function:

$$
\begin{aligned}
f_{i}(t)= & -(1-\kappa) \sum_{j=1}^{N} a_{i j}\left(\hat{x}_{i}-\widehat{x}_{j}\right)^{T} W\left(\hat{x}_{i}-\widehat{x}_{j}\right) \\
& +\lambda_{\max }(W)\left\|e_{i}\right\|^{2} \sum_{j=1}^{N}\left(4 a_{i j}+2 a_{i 0}\right) \geq 0,
\end{aligned}
$$

where $0<\kappa<1, W=P B B^{T} P$. According to the definition of measurement error and $\hat{x}_{i}(t)=x_{i}(t)-x_{0}(t)$, (35) can be rewritten as

$$
\begin{aligned}
u_{i}(t)= & -K \sum_{j=0}^{N} a_{i j}\left(x_{i}(t)-x_{j}(t)+e_{i}(t)-e_{j}(t)\right) \\
= & -K \sum_{j=0}^{N} a_{i j}\left(x_{i}(t)-x_{0}(t)-\left(x_{j}(t)-x_{0}(t)\right)\right) \\
& -K \sum_{j=0}^{N} a_{i j}\left(e_{i}(t)-e_{j}(t)\right) \\
= & -K \sum_{j=1}^{N} a_{i j}\left(\hat{x}_{i}(t)-\hat{x}_{j}(t)+e_{i}(t)-e_{j}(t)\right) \\
& -K a_{i 0}\left(\hat{x}_{i}(t)+e_{i}(t)\right) .
\end{aligned}
$$

Combining (2), (3), with (37) yields

$$
\dot{\hat{x}}(t)=\left(I_{N} \otimes A\right) \hat{x}(t)-((L+\bar{D}) \otimes B K)(\widehat{x}(t)+e(t)) \text {. }
$$

Theorem 3. Under Assumption 1, the multiagent systems (3) with protocol (35) can track system (2) successfully under the event-triggering condition (36), where $K=B^{T} P$ and $W=P B B^{T} P$. 
Proof. Define the Lyapunov function

$$
V_{2}=\frac{1}{2} \sum_{i=1}^{N} \widehat{x}_{i}^{T} P \widehat{x}_{i}
$$

Following the same proof as that of Theorem 1, the time derivation of $V_{2}$ along the trajectory of system (38) is obtained:

$$
\begin{aligned}
& \dot{V}_{2} \leq \sum_{i=1}^{N} \widehat{x}_{i}^{T} P A \widehat{x}_{i}-\frac{1}{2} \sum_{i=1}^{N} \sum_{j=1}^{N} a_{i j} \widehat{x}_{i}^{T} W\left(\widehat{x}_{i}-\widehat{x}_{j}\right) \\
& -\frac{1}{2} \sum_{i=1}^{N} \widehat{x}_{i}^{T} W a_{i 0} \widehat{x}_{i}+\frac{1}{2} \sum_{i=1}^{N} e_{i}^{T} W a_{i 0} e_{i} \\
& +\sum_{i=1}^{N} \sum_{j=1}^{N} a_{i j} e_{i}^{T} W e_{i} \\
& \leq \widehat{x}^{T}\left(I_{N} \otimes P A\right) \widehat{x}-\frac{1}{4} \sum_{i=1}^{N} \sum_{j=1}^{N} a_{i j}\left(\widehat{x}_{i}-\hat{x}_{j}\right)^{T} W\left(\hat{x}_{i}-\widehat{x}_{j}\right) \\
& -\frac{1}{2} \sum_{i=1}^{N} \widehat{x}_{i}^{T} W a_{i 0} \widehat{x}_{i}+\sum_{i=1}^{N}\left(\sum_{j=1}^{N} a_{i j} e_{i}^{T} W e_{i}+\frac{1}{2} e_{i}^{T} W a_{i 0} e_{i}\right) \\
& \leq \widehat{x}^{T}\left(I_{N} \otimes P A\right) \widehat{x}+\frac{1}{4} \sum_{i=1}^{N} \sum_{j=1}^{N} e_{i}^{T}\left(4 a_{i j}+2 a_{i 0}\right) W e_{i} \\
& -\frac{1}{4} \sum_{i=1}^{N} \sum_{j=1}^{N} a_{i j}\left(\hat{x}_{i}-\hat{x}_{j}\right)^{T} W\left(\hat{x}_{i}-\hat{x}_{j}\right) \\
& \leq \hat{x}^{T}\left(I_{N} \otimes\left(P A+A^{T} P\right)\right) \hat{x} \\
& +\frac{1}{4} \sum_{i=1}^{N} \lambda_{\max }(W)\left\|e_{i}\right\|^{2} \sum_{j=1}^{N}\left(4 a_{i j}+2 a_{i 0}\right) \\
& -\frac{1}{4} \sum_{i=1}^{N} \sum_{j=1}^{N} a_{i j}\left(\hat{x}_{i}-\hat{x}_{j}\right)^{T} W\left(\hat{x}_{i}-\hat{x}_{j}\right) .
\end{aligned}
$$

According to (6) and event-triggering condition (36), we can find that

$$
\begin{aligned}
\dot{V}_{2} \leq & \frac{1}{2} \widehat{x}^{T}\left(I_{N} \otimes\left(P A+A^{T} P\right)\right) \widehat{x} \\
& -\frac{\kappa}{4} \sum_{j=1}^{N} \sum_{i=1}^{N} a_{i j}\left(\widehat{x}_{i}-\widehat{x}_{j}\right)^{T} W\left(\widehat{x}_{i}-\widehat{x}_{j}\right) \\
\leq & -\frac{\kappa}{2} \widehat{x}^{T}(L \otimes W) \widehat{x} \\
\leq & -\frac{\kappa}{2} \lambda_{\max }(L) \lambda_{\max }(W)\|\widehat{x}\|^{2} \\
& \leq 0
\end{aligned}
$$

It can be seen from the abovementioned formula that $V_{2}$ is not increasing; therefore,

$$
V_{2}(0) \geq V_{2}(t)=\frac{1}{2} \sum_{i=1}^{N} \widehat{x}_{i}(t)^{T} P \widehat{x}_{i}(t) \geq \frac{1}{2} \lambda_{\min }(P)\|\widehat{x}(t)\|^{2} .
$$

That is to say, $\|\widehat{x}(t)\| \leq \sqrt{2\left(V_{2}(0) / \lambda_{\min }(P)\right)}=0$. According to LaSalle's invariance principle, we can obtain that system (38) can achieve consensus, that is, $\lim _{t \rightarrow \infty} \widehat{x}_{i}=0$, which is equivalent to $\lim _{t \rightarrow \infty}\left\|x_{i}(t)-x_{0}(t)\right\|=0, i=1,2, \ldots, N$. The proof is completed.

Theorem 4. Under the conditions of Theorem 3, system (38) does not exhibit Zeno behavior. The interval between any two consecutive event-triggering instants of the system is not less than

$$
\frac{\left(\|A\|+\left\|B K\left(\left(L_{i}+a_{i 0}\right) \otimes I_{n}\right)\right\|\right)}{3} \times\left(\left(1+\left((1-\kappa) \frac{a_{i 0} \lambda_{\min }(W)}{\left(2 d_{i i}+a_{i 0}\right) \lambda_{\max }(W)}\right)^{1 / 2}\right)^{3}-1\right) .
$$

Proof. It is similar to the proof of Theorem 2. The event interval between $t_{k}^{i}$ and $t_{k+1}^{i}$ is $\left(\left\|e_{i}(t)\right\| /\left\|\hat{x}_{i}(t)\right\|\right)$ which grows from 0 to $\left((1-\kappa)\left(a_{i 0} \lambda_{\min }(W) /\left(2 d_{i i}+a_{i 0}\right) \lambda_{\max }(W)\right)\right)^{1 / 2}$. The time derivative of $\left(\left\|e_{i}(t)\right\| /\left\|\widehat{x}_{i}(t)\right\|\right)$ is 


$$
\begin{aligned}
\frac{d}{d t} \frac{\left\|e_{i}\right\|}{\left\|\hat{x}_{i}\right\|} & \| \frac{\left\|\dot{x}_{i}(t)\right\|}{\left\|\widehat{x}_{i}(t)\right\|}+\frac{\left\|\dot{x}_{i}(t)\right\|\left\|e_{i}(t)\right\|}{\left\|\hat{x}_{i}(t)\right\|^{2}} \\
= & \frac{\left\|\dot{\hat{x}}_{i}(t)\right\|}{\left\|\widehat{x}_{i}(t)\right\|}\left(1+\frac{\left\|e_{i}(t)\right\|}{\left\|\widehat{x}_{i}(t)\right\|}\right) \\
= & \left(\|A\|+\left\|B K\left(\left(L_{i}+a_{i 0}\right) \otimes I_{n}\right)\right\|\right)\left(1+\frac{\left\|e_{i}(t)\right\|}{\left\|\widehat{x}_{i}(t)\right\|}\right) \\
& +\frac{\left\|B K\left(\left(L_{i}+a_{i 0}\right) \otimes I_{n}\right)\right\|\left\|e_{i}\right\|}{\left\|\widehat{x}_{i}(t)\right\|}\left(1+\frac{\left\|e_{i}(t)\right\|}{\left\|\widehat{x}_{i}(t)\right\|}\right) \\
\leq & \left(\|A\|+\left\|B K\left(\left(L_{i}+a_{i 0}\right) \otimes I_{n}\right)\right\|\right)\left(1+\frac{\left\|e_{i}(t)\right\|}{\left\|\widehat{x}_{i}(t)\right\|}\right) \\
= & \left(\|A\|+\left\|B K\left(\left(L_{i}+a_{i 0}\right) \otimes I_{n}\right)\right\|\right)\left(1+\frac{\left\|e_{i}(t)\right\|}{\left\|\widehat{x}_{i}(t)\right\|}\right)^{2}, \\
& +\frac{\left(\|A\|+\left\|B K\left(\left(L_{i}+a_{i 0}\right) \otimes I_{n}\right)\right\|\right)\left\|e_{i}\right\|\left(1+\frac{\left\|e_{i}(t)\right\|}{\left\|\widehat{x}_{i}(t)\right\|}\right)}{\left\|\widehat{x}_{i}(t)\right\|}
\end{aligned}
$$

where $L_{i}$ is the row $i$ of the Laplace matrix $L$.

Let $z_{i}=\left(\left\|e_{i}(t)\right\| /\left\|\widehat{x}_{i}(t)\right\|\right)$, then

$$
\dot{z}_{i} \leq\left(\|A\|+\left\|B K\left(\left(L_{i}+a_{i 0}\right) \otimes I_{n}\right)\right\|\right)\left(1+z_{i}\right)^{2} .
$$

Consider that a nonnegative function $\psi\left(t, \psi_{0}\right)$ satisfies $\dot{\psi}=\left(\|A\|+\left\|B K\left(\left(L_{i}+a_{i 0}\right) \otimes I_{n}\right)\right\|\right)(1+\psi)^{2}$ and $\psi_{0}=0$, according to Lemma $3, z_{i} \leq \psi(t, 0)$. It can be seen from (36),

$$
\psi\left(\tau_{k}^{i}, 0\right)=\left((1-\kappa) \frac{a_{i 0} \lambda_{\min }(W)}{\left(2 d_{i i}+a_{i 0}\right) \lambda_{\max }(W)}\right)^{1 / 2} .
$$

Hence,

$$
\begin{aligned}
\tau_{k}^{i}= & \frac{\left(\|A\|+\left\|B K\left(\left(L_{i}+a_{i 0}\right) \otimes I_{n}\right)\right\|\right)}{3}\left((1+\psi(\tau))^{3}-1\right), \\
= & \frac{\left(\|A\|+\left\|B K\left(\left(L_{i}+a_{i 0}\right) \otimes I_{n}\right)\right\|\right)}{3} \\
& \times\left(\left(1+\left((1-\kappa) \frac{a_{i 0} \lambda_{\min }(W)}{\left(2 d_{i i}+a_{i 0}\right) \lambda_{\max }(W)}\right)^{1 / 2}\right)^{3}-1\right) .
\end{aligned}
$$

Similar to Theorem 2, that Zeno behavior that does not occur can be proved by contradiction, which is omitted here.

\section{Leader-Following Control of Multiagent Systems under Switching Topologies}

In this part, we consider the extended case that the interconnection network switches according to signal $\sigma(t)$ and is not connected all the time. It is worth noting that, unlike the fixed topology, the controller updates only when the event is triggered. In the switching topologies, the controller updates in the following two cases: (1) event-triggering instant. (2) Communication topology switching instant.

The control input of the $i$ th agent is defined as follows:

$$
\begin{aligned}
u_{i}(t)= & -K \sum_{j \in N_{i}(t)} a_{i j}(t)\left(x_{i}\left(t_{k}^{i}\right)-x_{j}\left(t_{k^{\prime}}^{i}\right)\right) \\
& -K a_{i 0}(t)\left(x_{i}\left(t_{k}^{i}\right)-x_{0}(t)\right),
\end{aligned}
$$

where $t \in\left[t_{k}^{i}, t_{k+1}^{i}\right)$. Different from control protocols (10) and (35), $N_{i}(t)$ and $a_{i j}(t)$ in (48) are changed under the switching topologies. Matrices $\bar{L}_{\sigma(t)}$ and $\bar{D}_{\sigma(t)}$ in $\overline{\mathscr{G}}_{\sigma(t)}$ represent Laplacian matrix and connection matrix between leader and agent, respectively. Switching signal $\sigma(t):[0, \infty) \longrightarrow \mathscr{P}$ is a piecewise continuous constant function, which is used to describe the switching law of communication topology. Also, $\left\{\overline{\mathscr{G}}_{\sigma(t)}: p \in \mathscr{P}\right\}$ is a set of graphs that are switched within a finite set $\mathscr{P}=\{1,2, \ldots\}$ in any finite time interval. Consider a nonempty and continuous infinite sequence $\left[t_{s}, t_{s+1}\right)$, where $k=0,1, \ldots$ and $t_{0}=0$. Suppose that $\overline{\mathscr{G}}_{\sigma(t)}$ is switched only at and remains unchanged in $t \in\left[t_{s}, t_{s+1}\right)$.

Remark 2. It should be noted that graph $\overline{\mathscr{G}}_{\sigma(t)}$ may be connected or unconnected in interval $\left[t_{s}, t_{s+1}\right)$.

By replacing the similar variables in Section 3.2, we can derive that

$$
\dot{\hat{x}}(t)=\left(I_{N} \otimes A\right) \widehat{x}(t)-\left(\left(L_{\sigma(t)}+\bar{D}_{\sigma(t)}\right) \otimes B K\right)(\widehat{x}(t)+e(t)) .
$$

Theorem 5. Under Assumptions 1 and 2, if feedback gain matrix $K$ satisfies $K=B^{T} P$ and $W=P B K$, then the protocol (48) still makes the multiagent system with (3) track the system (2) successfully if the event-triggering condition satisfies

$$
\begin{aligned}
f_{i}(t)= & -\kappa \frac{a_{i 0_{\min }} \lambda_{\min }(W)-\alpha \lambda_{\text {max }}(P)}{a_{i 0_{\max }} \lambda_{\max }(W)+2 d_{i i_{\max }} \lambda_{\max }(W)} \sum_{i=1}^{N} \widehat{x}_{i}^{T} \widehat{x}_{i} \\
& +\sum_{i=1}^{N} e_{i}^{T} e_{i} \geq 0,
\end{aligned}
$$

where $0<\kappa<1,0<\alpha \leq\left(a_{i 0_{\sigma(t)}} \lambda_{\min }(W) / \lambda_{\max }(P)\right)$.

Proof. Construct the Lyapunov function for system (49) as follows:

$$
V_{3}=e^{\alpha t} \sum_{i=1}^{N} \hat{x}_{i}^{T} P \widehat{x}_{i} .
$$

Similar to Section 3.2, taking the derivative of $V_{3}$ along the trajectory of system (49) yields 


$$
\begin{aligned}
\dot{V}_{3} \leq & 2 e^{\alpha t} \sum_{i=1}^{N} \hat{x}_{i}^{T} P A \widehat{x}_{i}-e^{\alpha t} \sum_{i=1}^{N} \sum_{j=1}^{N} a_{i j} \widehat{x}_{i}^{T} W\left(\widehat{x}_{i}-\widehat{x}_{j}\right) \\
& +e^{\alpha t} \sum_{i=1}^{N} e_{i}^{T} W a_{i 0} e_{i}+2 e^{\alpha t} \sum_{i=1}^{N} \sum_{j=1}^{N} a_{i j} e_{i}^{T} W e_{i} \\
& +e^{\alpha t} \sum_{i=1}^{N} \hat{x}_{i}^{T} \alpha P \widehat{x}_{i}-e^{\alpha t} \sum_{i=1}^{N} \widehat{x}_{i}^{T} W a_{i 0} \widehat{x}_{i} \\
\leq & e^{\alpha t} \widehat{x}^{T}\left(I_{N} \otimes\left(P A+A^{T} P\right)\right) \hat{x}-e^{\alpha t} \widehat{x}^{T}\left(L_{\sigma(t)} \otimes W\right) \widehat{x} \\
& +e^{\alpha t} \sum_{i=1}^{N} e_{i}^{T}\left(a_{i 0_{\sigma(t)}} W+2 d_{i i_{\sigma(t)}} W\right) e_{i} \\
& +e^{\alpha t} \sum_{i=1}^{N} \hat{x}_{i}^{T}\left(\alpha P-a_{i 0_{\sigma(t)}} W\right) \widehat{x}_{i} .
\end{aligned}
$$

(i) If the graph $\overline{\mathscr{G}}_{p}$ is not connected during $t \in\left[t_{s}, t_{s+1}\right)$, according to the event-triggering condition (50) and equation (6), one has

$$
\begin{aligned}
\dot{V}_{3} \leq & e^{\alpha t} \widehat{x}^{T}\left(I_{N} \otimes\left(P A+A^{T} P\right)\right) \hat{x} \\
& +e^{\alpha t} \sum_{i=1}^{N}\left(\alpha \lambda_{\max }(P)-a_{i 0_{\sigma(t)}} \lambda_{\min }(W)\right)\left\|\hat{x}_{i}\right\|^{2} \\
& +e^{\alpha t} \sum_{i=1}^{N}\left(a_{i 0_{\sigma(t)}} \lambda_{\max }(W)+2 d_{i i_{\sigma(t)}} \lambda_{\max }(W)\right)\left\|e_{i}\right\|^{2}
\end{aligned}
$$

$\leq 0$.

It can be seen from the abovementioned formula that $V_{3}$ is not increasing; hence,

$$
\begin{aligned}
V_{3}(t) \geq V_{3}\left(t_{s+1}\right) & =e^{\alpha t_{s+1}} \sum_{i=1}^{N} \hat{x}_{i}\left(t_{s+1}\right)^{T} P \widehat{x}_{i}\left(t_{s+1}\right) \\
& \geq e^{\alpha t_{s+1}} \lambda_{\min }(P)\left\|\hat{x}\left(t_{s+1}\right)\right\|^{2},
\end{aligned}
$$

i.e., $\sqrt{\left(V_{3}(0) / \lambda_{\min }(P)\right)} e^{-(\alpha / 2) t_{s+1}}$.

$\left\|\widehat{x}\left(t_{s+1}\right)\right\| \leq \sqrt{\left(V_{3}(t) / \lambda_{\min }(P)\right)} e^{-(\alpha / 2) t_{s+1}} \leq$

(ii) If the graph $\overline{\mathscr{G}}_{p}$ is connected during $t \in\left[t_{s}, t_{s+1}\right)$, then

$$
\begin{aligned}
\dot{V}_{3} \leq e^{\alpha t} \widehat{x}^{T}\left(I_{N} \otimes\left(P A+A^{T} P-\lambda_{2}\left(L_{\sigma(t)}\right) W\right)\right) \hat{x} \\
+e^{\alpha t} \sum_{i=1}^{N}\left(\alpha \lambda_{\max }(P)-a_{i 0_{\sigma(t)}} \lambda_{\min }(W)\right)\left\|\hat{x}_{i}\right\|^{2} \\
+e^{\alpha t} \sum_{i=1}^{N}\left(a_{i 0_{\sigma(t)}} \lambda_{\max }(W)+2 d_{i i_{\sigma(t)}} \lambda_{\max }(W)\right)\left\|e_{i}\right\|^{2} .
\end{aligned}
$$

According to event-triggering condition (50) and equation (5),

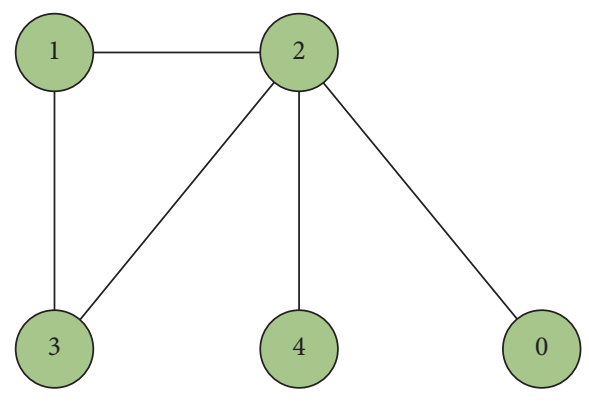

Figure 1: Communication topology $\overline{\mathscr{G}}$.
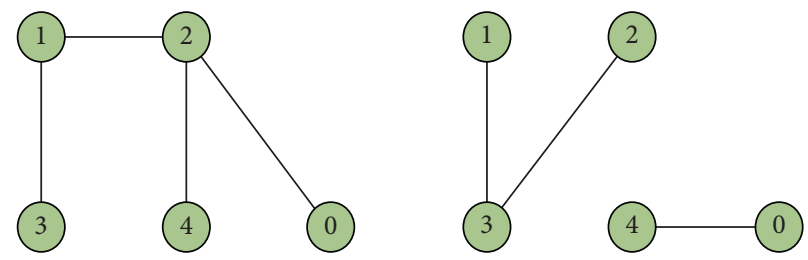

Figure 2: Communication topology $\overline{\mathscr{G}}_{1}$ and $\overline{\mathscr{G}}_{2}$.

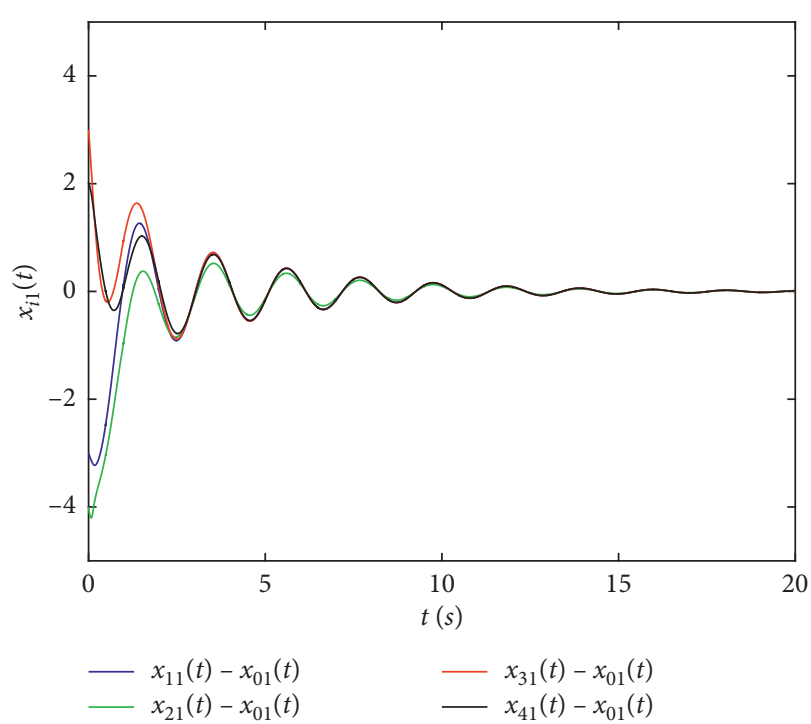

FIGURE 3: The 1st state error trajectory of each agent under protocol (10).

$$
\begin{aligned}
\dot{V}_{3} & \leq e^{\alpha t} \widehat{x}^{T}\left(I_{N} \otimes\left(P A+A^{T} P-\lambda_{2}\left(L_{\sigma(t)}\right) W\right)\right) \hat{x} \\
& \leq-e^{\alpha(t)} \frac{\lambda_{2}\left(L_{\sigma(t)}\right)}{2} \widehat{x}^{T} \hat{x} .
\end{aligned}
$$

It can be seen from (56) that $V_{3}$ is not increasing; hence,

$$
\begin{aligned}
V_{3}(t) & \geq V_{3}\left(t_{s+1}\right)=e^{\alpha t_{s+1}} \sum_{i=1}^{N} \widehat{x}_{i}\left(t_{s+1}\right)^{T} P \widehat{x}_{i}\left(t_{s+1}\right) \\
& \geq e^{\alpha t_{s+1}} \lambda_{\min }(P)\left\|\hat{x}\left(t_{s+1}\right)\right\|^{2}
\end{aligned}
$$

i.e., $\sqrt{\left(V_{3}(0) / \lambda_{\min }(P)\right)} e^{-(\alpha / 2) t_{s+1}}$. 


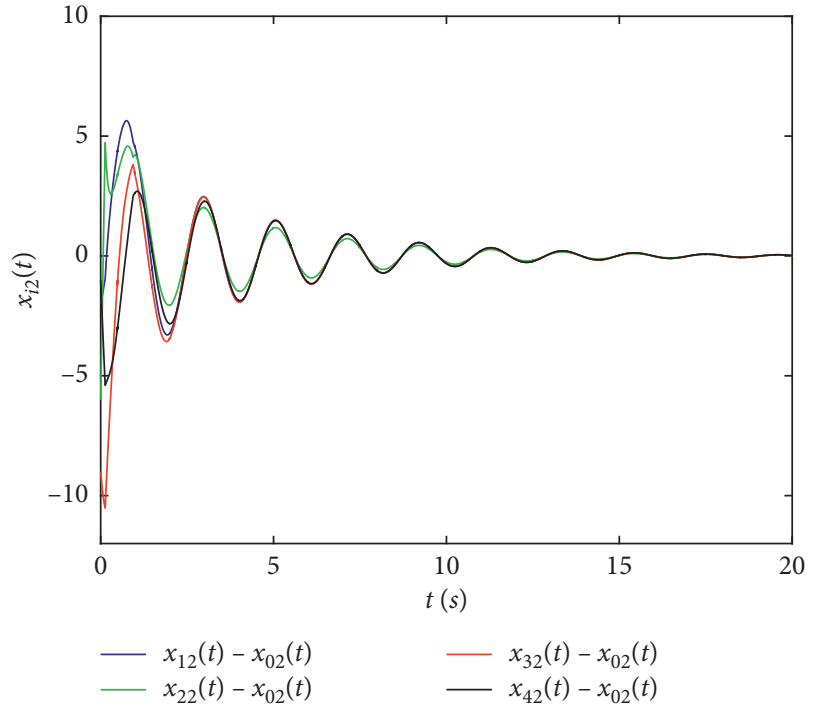

FIGURE 4: The 2st state error trajectory of each agent under protocol (10).

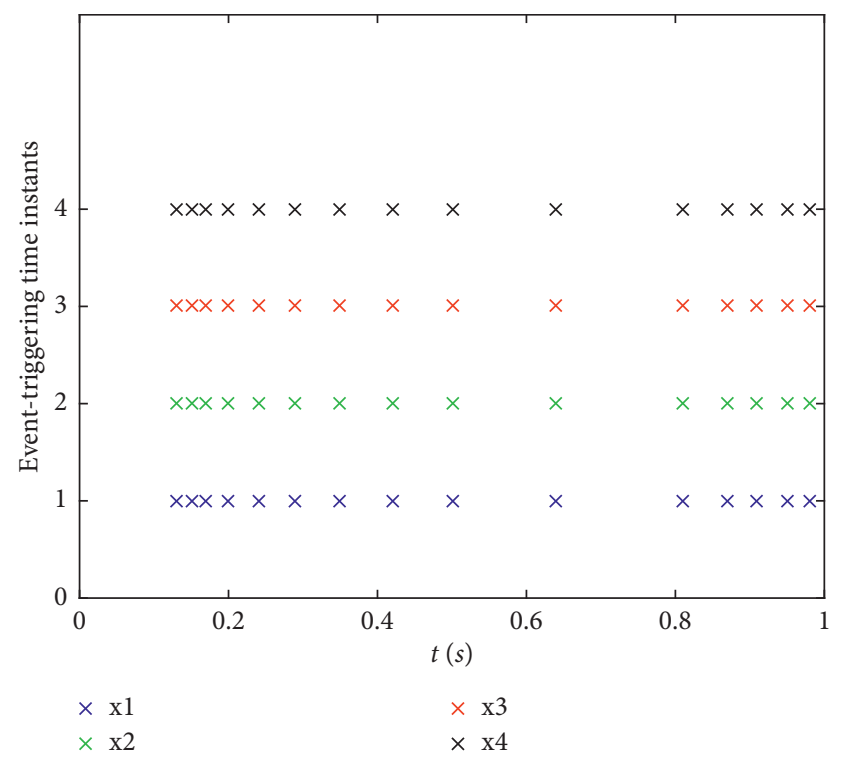

FIgURE 5: Event times instants for four agents in Theorem 1.

In summary, $\quad\left\|\widehat{x}\left(t_{s+n}\right)\right\| \leq \sqrt{\left(V_{3}\left(t_{s+(n-1)}\right) / \lambda_{\min }(P)\right)}$ $e^{-(\alpha / 2) t_{s+n}} \leq \cdots \leq \sqrt{\left(V_{3}(0) / \lambda_{\min }(P)\right)} e^{-(\alpha / 2) t_{s+n}}$, i.e., $\|\hat{x}(t)\| \leq$ $\sqrt{\left(V_{3}(t) / \lambda_{\min }(P)\right)} e^{-(\alpha / 2) t} \leq \cdots \leq \sqrt{\left(V_{3}(0) / \lambda_{\min }(P)\right)} e^{-(\alpha / 2) t}$, so $\lim _{t \rightarrow \infty}\|\widehat{x}(t)\|=0$ is equivalent to $\lim _{t \rightarrow \infty}\left\|\widehat{x}_{i}(t)\right\|=0$, and accordingly $\lim _{t \rightarrow \infty}\left\|x_{i}(t)-x_{0}(t)\right\|=0, i=1,2, \ldots, N$ is established.

Remark 3. Index $(\alpha / 2)$ can be approximated as the convergence rate of multiagent system (49), and the convergence rate can be changed by adjusting $\alpha$.

Theorem 6. Under the conditions of Theorem 5, system (49) does not have Zeno behavior. The interval between any two

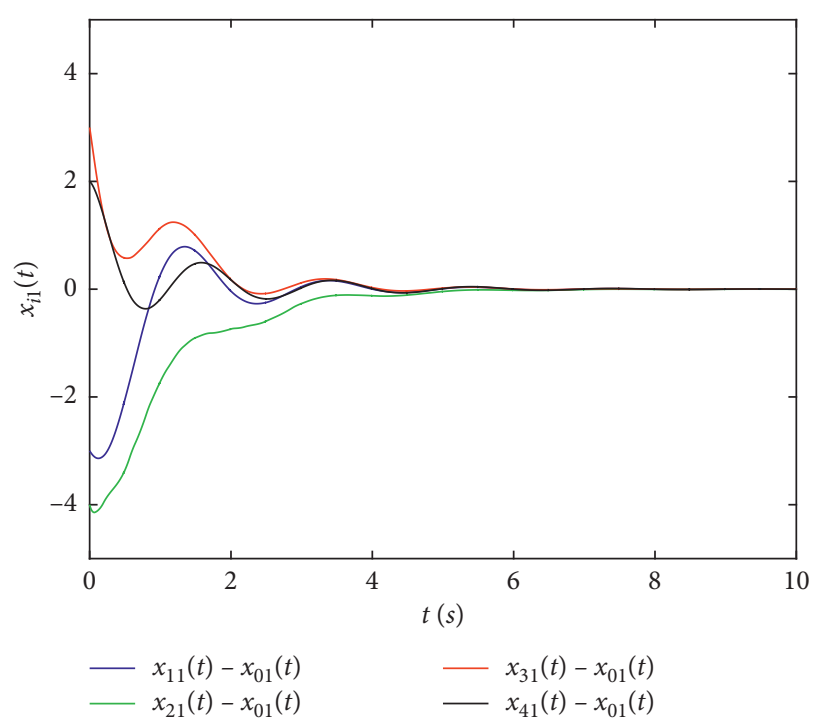

FIGURE 6: The 1st state error trajectory of each agent under protocol (35).

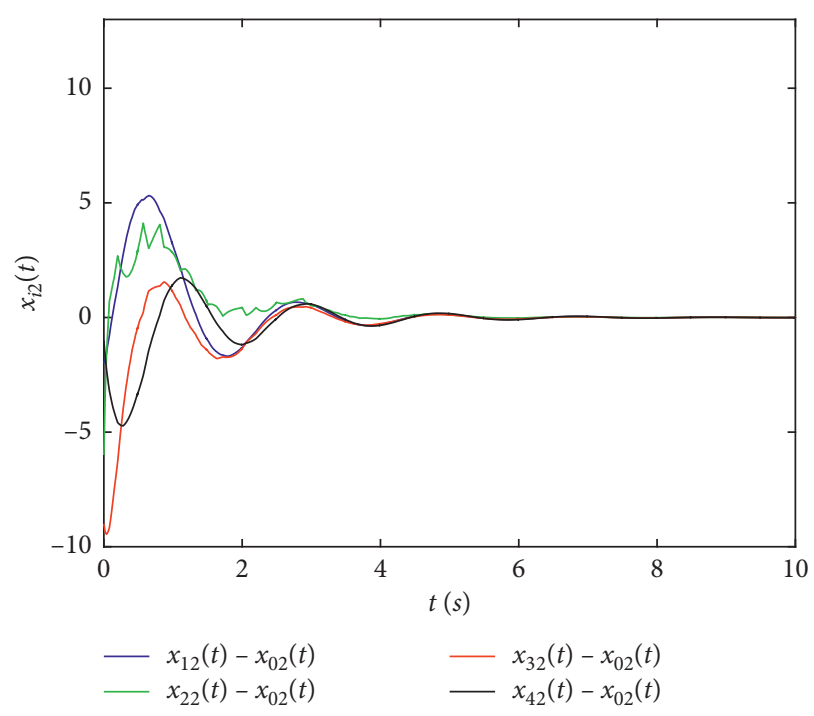

Figure 7: The 2nd state error trajectory of each agent under protocol (35).

consecutive event-triggering instants of the system is not less than

$$
\begin{aligned}
& \frac{\left(\left\|I_{N} \otimes A\right\|+\|(L+\bar{D}) \otimes B K\|\right)}{3} \\
& \quad \times\left(\left(1+\left(\frac{\kappa\left(a_{i 0_{\sigma(t)}} \lambda_{\min }(W)-\alpha \lambda_{\max }(P)\right)}{a_{i 0_{\sigma(t)}} \lambda_{\max }(W)+2 d_{i i_{\sigma(t)}} \lambda_{\max }(W)}\right)^{1 / 2}\right)^{3}-1\right) .
\end{aligned}
$$

Proof. The proof is similar to that of Theorem 2. 


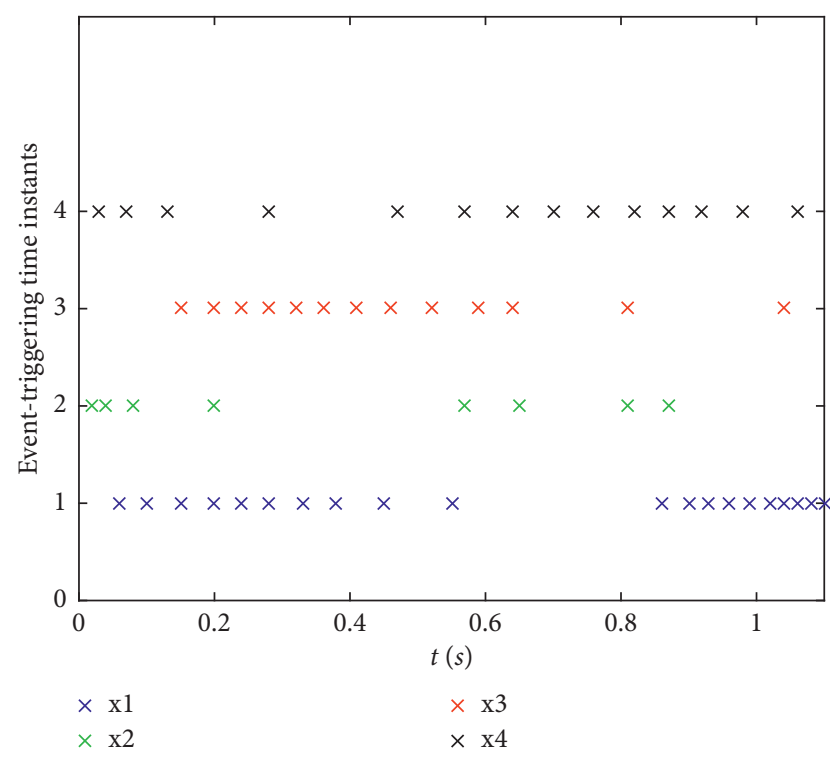

Figure 8: Event times instants for four agents in Theorem 3.

\section{Simulation}

In this part, we consider the trajectories of the state errors between the follower and leader under the fixed topology and the switching topology, respectively, where the dynamic equations of the leader and the follower are given by (2) and (3), respectively, and the communication network topology among agents is shown in Figures 1 and 2. Assume that $x_{i}=\left[x_{i 1}, x_{i 2}\right]^{T}$, and $A$ and $B$ are chosen as follows: $A=\left[\begin{array}{cc}0 & 0.5 \\ -4.8 & 0\end{array}\right], B=\left[\begin{array}{c}0 \\ -0.5\end{array}\right]$, it is easy to prove that the Assumption 2 is satisfied. By solving Riccati equation by MATLAB, we know that feedback gain matrix $K=B^{T} P=$ $[-0.4995,-1.1343]^{T}$. Let the leader's initial state be $x_{0}(0)=$ $[2,3]^{T}$ and the follower's initial state be $x_{1}(0)=[-1,1]^{T}$, $x_{2}(0)=[-2,-3]^{T}, x_{3}(0)=[5,-6]^{T}, x_{4}(0)=[4,2]^{T}$.

Example 1. Under the centralized event-triggering protocol (10), the leader-following consensus of the multiagent system composed of (2) and (3) is considered. The communication network among agents is shown in Figure 1 , and the corresponding weights are all 1 . It can be seen from Figures 3 and 4 that followers can successfully follow the leader. Figure 5 shows the event instants of each follower with the centralized event-triggering protocol (10). It can be seen that protocol (10) can effectively reduce the number of communications among agents, thus reducing the waste of resources. Also, there is no Zeno behavior.

Example 2. In this example, we illustrate the leader-following consensus of the multiagent system under the distributed event-triggering protocol (35). The communication network among agents is shown in Figure 1. It can be seen from Figures 6 and 7 that followers can successfully follow the leader. Figure 8 shows the event triggering time of each follower under the decentralized event triggering protocol

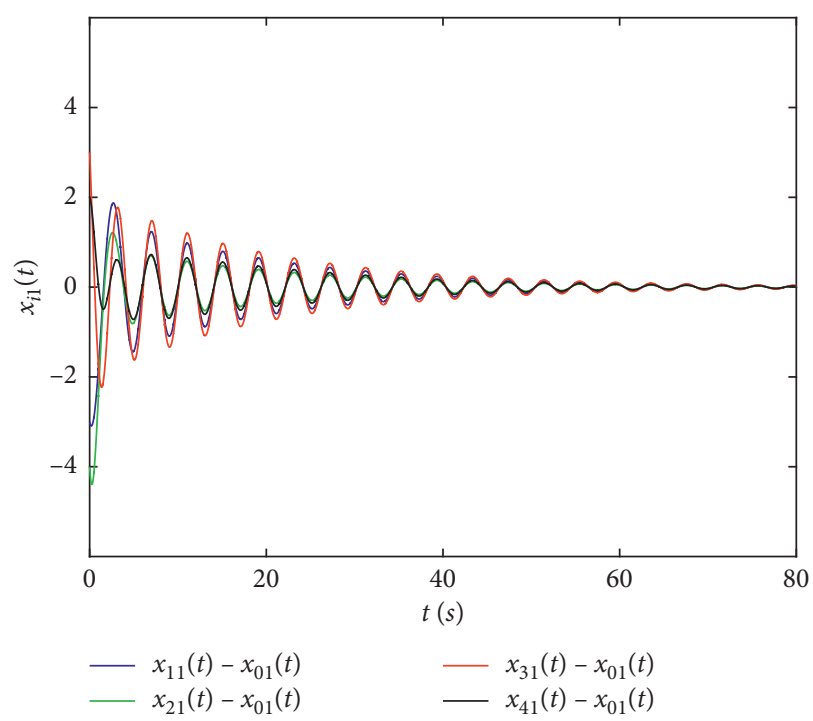

FIGURE 9: The 1st state error trajectory of each agent under protocol (48).

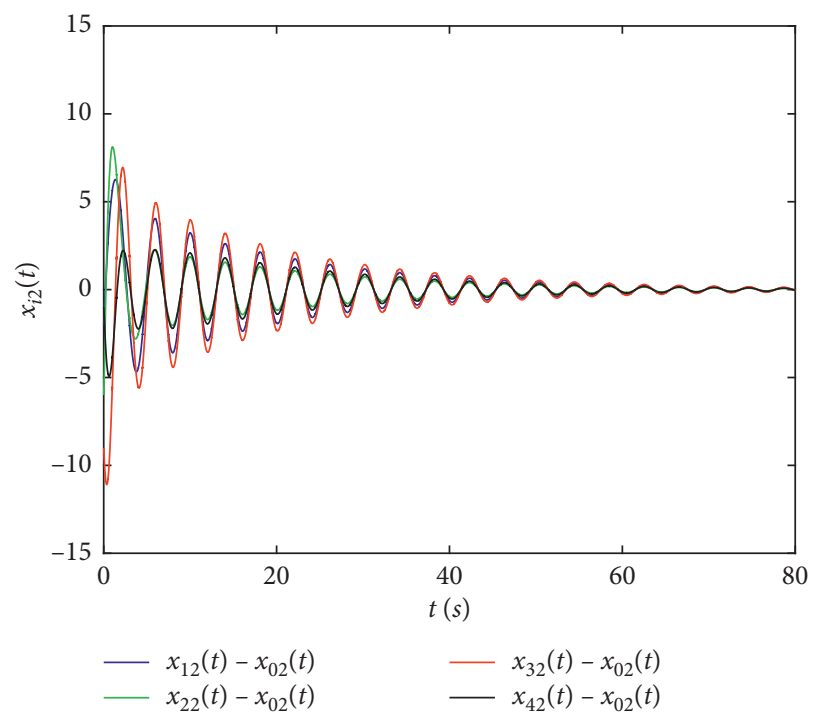

FIGURE 10: The 2nd state error trajectory of each agent under protocol (48).

(35), and Zeno behavior is excluded. The simulation results verify Theorems 3 and 4 .

Example 3. Finally, the leader-following consensus of the multiagent system under the control protocol (48) is considered. The communication network among agents will randomly switch between $\overline{\mathscr{G}}_{1}$ and $\overline{\mathscr{G}}_{2}$, as shown in Figure 2, where $\overline{\mathscr{G}}_{1}$ is a connected graph and $\overline{\mathscr{G}}_{2}$ is an unconnected graph. The state errors between the follower agent $i$ and leader 0 are shown in Figures 9 and 10, respectively. It indicates that all followers can successfully follow the leader. Figure 11 shows the event-triggering instants of each follower under (48), and there is no Zeno behavior. 


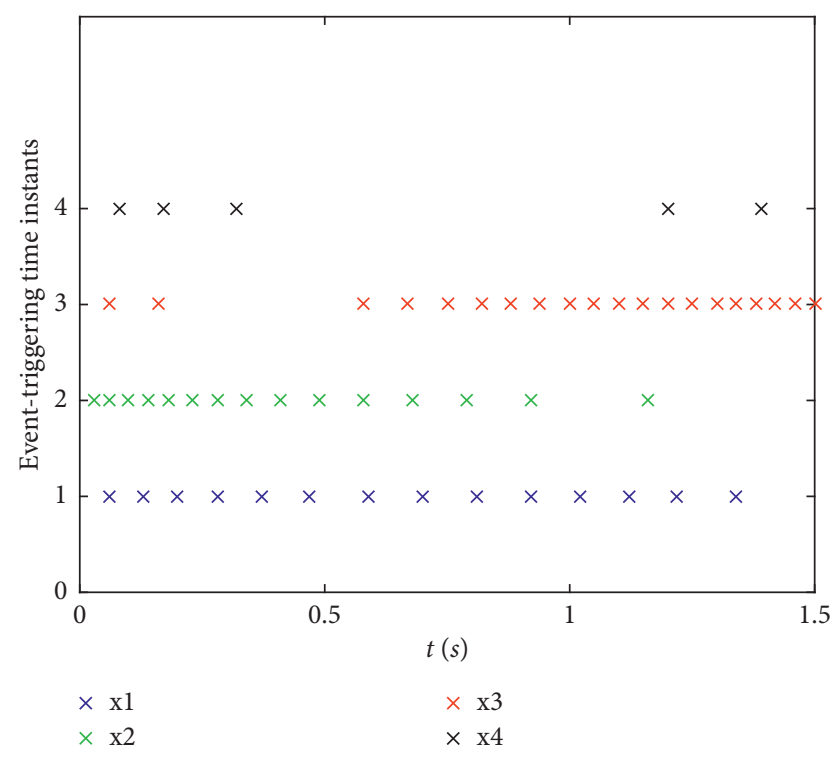

Figure 11: Event times instants for four agents in Theorem 5.

\section{Conclusions and Future Work}

In this paper, the leader-following control of general linear multiagent systems based on event-triggering mechanism under both fixed topology and switching topologies have been studied. Under the fixed topology, two different control protocols are designed in order to reduce waste of resources. Based on these two control protocols, we propose two different triggering functions, i.e., centralized event-triggering function and decentralized event-triggering function with state error between the follower and leader. When the triggering function exceeds 0 , the agent will update the control input at the triggering instants. Through theoretical analysis, the sufficient conditions are derived for the system to achieve leader-following consensus under two control protocols and event-triggering conditions. The conditions obtained under fixed topology are extended to switching topologies (different from the fixed topology, the controller update at the triggering time, and also the switching time). The results show that the conditions to achieve leader-following are also valid under switching topologies. Finally, it is proved that the system can realize leader-following control without Zeno behavior. The simulation results verify the effectiveness of the theoretical analysis. In the future, we will further study the leader-following control of the linear multiagent system with interference, delay, and other factors.

\section{Data Availability}

No data were used to support this study.

\section{Conflicts of Interest}

The authors declare that they have no conflicts of interest.

\section{Acknowledgments}

This work was supported by the National Natural Science Foundation of China (Grant.no. 61873136, 61903210, 61374062, and 61603288); Science Foundation of Shandong Province for Distinguished Young Scholars (Grant.no. JQ201419); and Shandong Provincial Natural Science Foundation, China (Grant.no. ZR201709260010).

\section{References}

[1] D. Meng, "Dynamic distributed control for networks with cooperative-antagonistic interactions," IEEE Transactions on Automatic Control, vol. 63, no. 8, pp. 2311-2326, 2018.

[2] D. Meng, "Bipartite containment tracking of signed networks," Automatica, vol. 79, pp. 282-289, 2017.

[3] X. Liu, Z. Ji, and T. Hou, "Graph partitions and the controllability of directed signed networks," Science China Information Sciences, vol. 62, no. 4, Article ID 42202, 2019.

[4] Y. Chao and Z. Ji, "Necessary and sufficient conditions for multi-agent controllability of path and star topologies by exploring the information of second-order neighbours," IMA Journal of Mathematical Control and Information, 2016.

[5] X. Liu and Z. Ji, "Controllability of multiagent systems based on path and cycle graphs," International Journal of Robust and Nonlinear Control, vol. 28, no. 1, pp. 296-309, 2018.

[6] Z. Ji, H. Lin, and H. Yu, "Protocols design and uncontrollable topologies construction for multi-agent networks," IEEE Transactions on Automatic Control, vol. 60, no. 3, pp. 781-786, 2015.

[7] Z. Ji and H. Yu, "A new perspective to graphical characterization of multiagent controllability," IEEE Transactions on Cybernetics, vol. 47, no. 6, pp. 1471-1483, 2017.

[8] N. Cai, M. He, Q. Wu, and M. J. Khan, "On almost controllability of dynamical complex networks with noises," Journal of Systems Science and Complexity, vol. 32, no. 4, pp. 1125-1139, 2019.

[9] Y. Guan, Z. Ji, L. Zhang, and L. Wang, "Controllability of multi-agent systems under directed topology," International Journal of Robust and Nonlinear Control, vol. 27, no. 18, pp. 4333-4347, 2017.

[10] Y. Guan, Z. Ji, L. Zhang, and L. Wang, "Controllability of heterogeneous multi-agent systems under directed and weighted topology," International Journal of Control, vol. 89, no. 5, pp. 1009-1024, 2016.

[11] Z. Lu, L. Zhang, Z. Ji, and L. Wang, "Controllability of discrete-time multiagent systems with directed topology and input delay," International Journal of Control, vol. 89, no. 1, pp. 179-192, 2016.

[12] X. Liu, Z. Ji, and T. Hou, "Stabilization of heterogeneous multi-agent systems via harmonic control," Complexity, vol. 2018, Article ID 8265637, 9 pages, 2018.

[13] K. Liu, Z. Ji, and W. Ren, "Necessary and sufficient conditions for consensus of second-order multi-agent systems under directed topologies without global gain dependency," IEEE Transactions on Cybernetics, vol. 47, no. 8, pp. 2089-2098, 2017.

[14] J. Qu, Z. Ji, C. Lin, and H. Yu, "Fast consensus seeking on networks with antagonistic interactions," Complexity, vol. 2018, Article ID 7831317, 15 pages, 2018. 
[15] J. Xi, C. Wang, X. Yang, and B. Yang, "Limited-budget output consensus for descriptor multiagent systems with energy constraints," IEEE Transactions on Cybernetics, pp. 21682275, 2020, http://arxiv.org/abs/1909.08345.

[16] Q. Qi, H. Zhang, and Z. Wu, "Stabilization control for linear continuous-time mean-field systems," IEEE Transactions on Automatic Control, vol. 64, no. 9, pp. 3461-3468, 2019.

[17] L. Tian, Z. Ji, T. Hou, and K. Liu, "Bipartite consensus on coopetition networks with time-varying delays," IEEE Access, vol. 6, no. 1, pp. 10169-10178, 2018.

[18] J. Xi, M. He, H. Liu, and J. Zheng, "Admissible output consensualization control for singular multi-agent systems with time delays," Journal of the Franklin Institute, vol. 353, no. 16, pp. 4074-4090, 2016.

[19] R. Olfati-Saber and R. M. Murray, "Consensus problems in networks of agents with switching topology and time-delays," IEEE Transactions on Automatic Control, vol. 49, no. 9, pp. 1520-1533, 2004.

[20] L. Wang, J. Xi, M. He, and G. Liu, "Robust time-varying formation design for multi-agent systems with disturbances: extended-state-observer method," International Journal of Robust and Nonlinear Control, http://arxiv.org/abs/1909. 08974, 2019.

[21] K. Liu and Z. Ji, "Consensus of multi-agent systems with time delay based on periodic sample and event hybrid control," Neurocomputing, vol. 270, pp. 11-17, 2017.

[22] H. Liu, G. Xie, and L. Wang, "Necessary and sufficient conditions for solving consensus problems of double-integrator dynamics via sampled control," International Journal of Robust and Nonlinear Control, vol. 20, no. 15, pp. 1706-1722, 2010.

[23] Y. Gao, B. Liu, J. Yu, J. Ma, and T. Jiang, "Consensus of firstorder multi-agent systems with intermittent interaction," Neurocomputing, vol. 129, pp. 273-278, 2014.

[24] P. Tabuada, "Event-triggered real-time scheduling of stabilizing control tasks," IEEE Transactions on Automatic Control, vol. 52, no. 9, pp. 1680-1685, 2007.

[25] D. V. Dimarogonas and E. Frazzoli, "Distributed eventtriggered control strategies for multi-agent systems," in Proceeings of the 2009 47th Annual Allerton Conference on Communication, Control, and Computing, IEEE, Monticello, IL, USA, October 2009.

[26] D. V. Dimarogonas, E. Frazzoli, and K. H. Johansson, "Distributed event-triggered control for multi-agent systems," IEEE Transactions on Automatic Control, vol. 57, no. 5, pp. 1291-1297, 2012.

[27] H. Yan, Y. Shen, H. Zhang, and H. Shi, "Decentralized eventtriggered consensus control for second-order multi-agent systems," Neurocomputing, vol. 133, no. 8, pp. 18-24, 2014.

[28] Y. Fan, G. Feng, Y. Wang, and C. Song, "Distributed eventtriggered control of multi-agent systems with combinational measurements," Automatica, vol. 49, no. 2, pp. 671-675, 2013.

[29] J. Hu, G. Chen, and H. Li, "Distributed event-triggered tracking control of second-order leader-follower multi-agent systems," in Proceeedings of the 30th Chinese Control Conference, Yantai, China, July 2011.

[30] H. Li, X. Liao, T. Huang, and W. Zhu, "Event-Triggering sampling based leader-following consensus in second-order multi-agent systems," IEEE Transactions on Automatic Control, vol. 60, no. 7, pp. 1998-2003, 2015.

[31] D. Xie, S. Xu, Y. Zou, and Z. Li, "Event-triggered consensus control for second-order multi-agent systems," IET Control Theory \& Applications, vol. 9, no. 5, pp. 667-680, 2015.
[32] D. Xie, S. Xu, Y. Chu, and Y. Zou, "Event-triggered average consensus for multi-agent systems with nonlinear dynamics and switching topology," Journal of the Franklin Institute, vol. 352, no. 3, pp. 1080-1098, 2015.

[33] G. S. Seyboth, D. V. Dimarogonas, and K. H. Johansson, "Event-based broadcasting for multi-agent average consensus," Automatica, vol. 49, no. 1, pp. 245-252, 2013.

[34] X. Meng and T. Chen, "Event based agreement protocols for multi-agent networks," Automatica, vol. 49, no. 7, pp. 21252132, 2013.

[35] F. Xiao, X. Meng, and T. Chen, "Average sampled-data consensus driven by edge events," in Proceedings of the Chinese Control Conference (CCC), pp. 6239-6244, Hefei, China, July 2012.

[36] Z. Zhang and L. Wang, "Distributed integral-type eventtriggered synchronization of multi-agent systems," International Journal of Robust and Nonlinear Control, vol. 28, no. 14, pp. 4175-4187, 2018.

[37] Z. Zhang, F. Hao, L. Zhang, and L. Wang, "Consensus of linear multi-agent systems via event-triggered control," International Journal of Control, vol. 87, no. 6, pp. 1243-1251, 2014.

[38] W. Hu, L. Liu, and G. Feng, "Leader-following consensus of linear multi-agent systems by distributed event-triggered control," in Proceedings of the 34th Chinese Control Conference, Hangzhou, China, July 2015.

[39] W. Zhu, Z.-P. Jiang, and G. Feng, "Event-based consensus of multi-agent Systems with general linear models," Automatica, vol. 50, no. 2, pp. 552-558, 2014.

[40] C. Nowzari and J. Cortés, "Distributed event-triggered coordination for average consensus on weight-balanced digraphs," Automatica, vol. 68, no. 4, pp. 237-244, 2016.

[41] Z. Li and Z. Duan, $H_{\infty}$ Cooperative Control of Multi-Agent Systems: A Consensus Region Approach, CRC Press, Boca raton, FL, USA, 2014. 